Supplement of Geosci. Instrum. Method. Data Syst., 9, 317-336, 2020

https://doi.org/10.5194/gi-9-317-2020-supplement

(c) Author(s) 2020. This work is distributed under

the Creative Commons Attribution 4.0 License.

(c) (1)

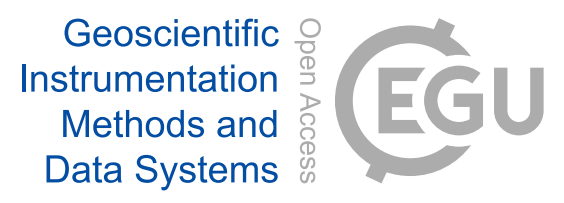

Supplement of

\title{
A monitoring system for spatiotemporal electrical self-potential measure- ments in cryospheric environments
}

Maximilian Weigand et al.

Correspondence to: Maximilian Weigand (mweigand@geo.uni-bonn.de)

The copyright of individual parts of the supplement might differ from the CC BY 4.0 License. 


\section{S1: Measurement flowchart}

\begin{tabular}{|c|c|c|}
\hline schedule & measured parameter & action \\
\hline $\begin{array}{l}\text { daily } \\
\text { hourly for } V_{\text {bat }}>12.3 \mathrm{~V} \\
\text { or } \\
6 \text {-hourly for } V_{\text {bat }}<12.2 \mathrm{~V}\end{array}$ & $\begin{array}{l}\text { contact resistances } \\
\text { electrode temperatures }\end{array}$ & $\begin{array}{l}\text { measure } \\
\text { measure }\end{array}$ \\
\hline $\begin{array}{l}\mathbf{5} \text { am - 8 pm } \\
10 \text { min, if } V_{\text {bat }}>12.6 \mathrm{~V} \\
\text { hourly, } \\
\text { if } V_{\text {bat }}>12.3 V_{;}<12.5 \mathrm{~V} \\
6 \text {-hourly, if } V_{\text {bat }}<12.2 \mathrm{~V}\end{array}$ & $\begin{array}{l}\text { SP-voltages } \\
\text { battery voltage } \\
\text { air-temperature } \\
\text { solar voltage }\end{array}$ & \\
\hline $\begin{array}{l}8 \mathbf{~ a m ~ - ~} \mathbf{~ p m} \\
30 \text { min, if } V_{\text {bat }}>12.6 \mathrm{~V} \\
\text { hourly, } \\
\text { if } V_{\text {bat }}>12.3 V_{;}<12.5 \mathrm{~V} \\
6 \text {-hourly, if } V_{\text {bat }}<12.2 \mathrm{~V}\end{array}$ & $\begin{array}{l}\text { SP-voltages } \\
\text { battery voltage } \\
\text { air-temperature } \\
\text { solar voltage }\end{array}$ & \\
\hline $\begin{array}{l}\text { 6-hourly } \\
\text { daily }\end{array}$ & & $\begin{array}{c}\text { send data by email } \\
\text { update logger time to UTC using ntp }\end{array}$ \\
\hline
\end{tabular}

Table 1: Measurement schedule of the measurement system. Note that data upload and ntp schedules have been varied over time to reduce power consumption and increase system reliability 


\section{S2: Internal signal flow}

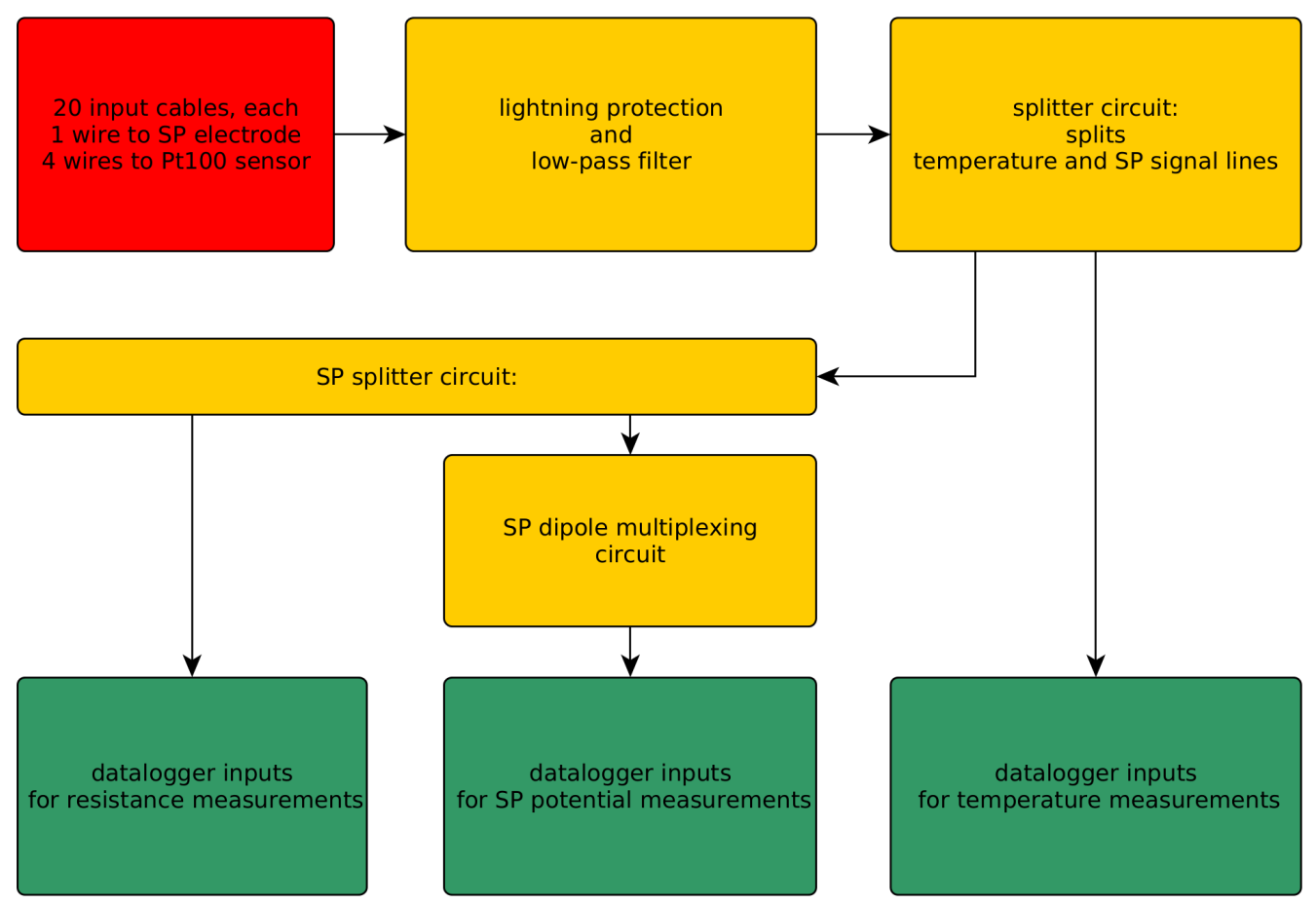

Figure 1: Signal flow in the measurement system. The incoming signal lines (upper left, red) are routed and multiplexed from the incoming cables using custom-made PCB boards, finally connecting to multiple data logger input channels (bottom, green). 


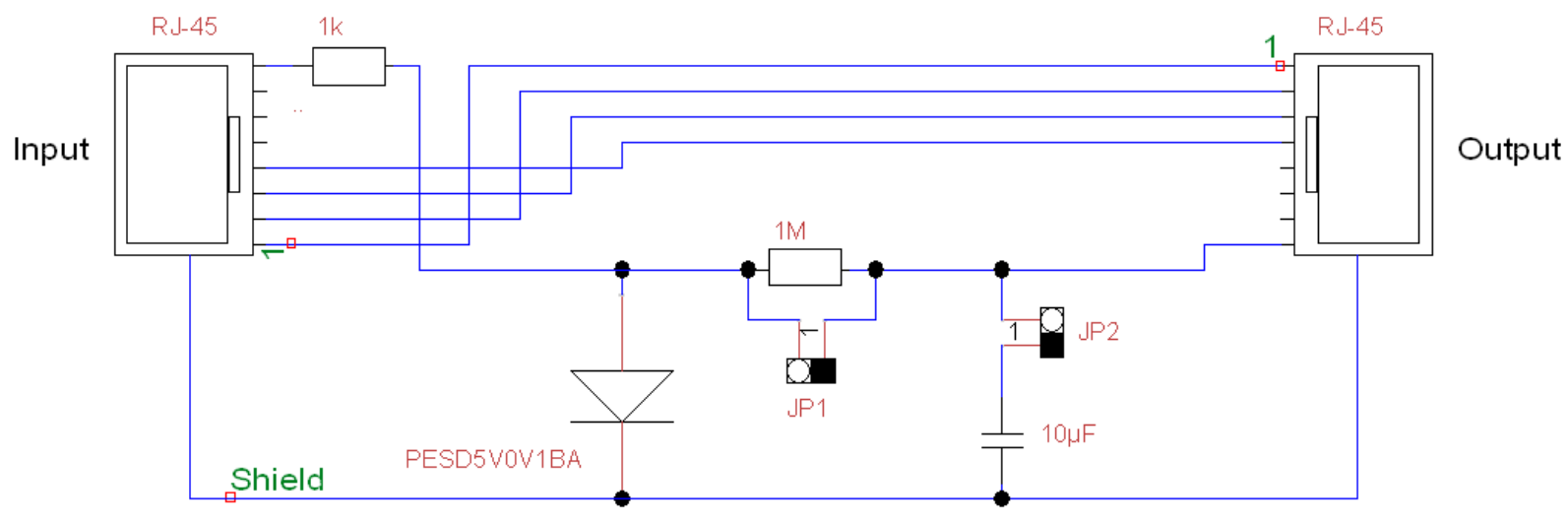

Figure 2: Lightning protection and low-pass circuit, applied to each SP electrode input line (as such this circuit was duplicated 20 times, using 4 pcb boards with 5 circuits each). The electrode and temperature signals arrive into the SP system via ethernet cables (one electrode group per cable), which are then connected to an ethernet panel (see Fig. 5 of manuscript, lower right corner). From here on each electrode group is routed using a short ethernet cable to to the lightning protection circuit. 


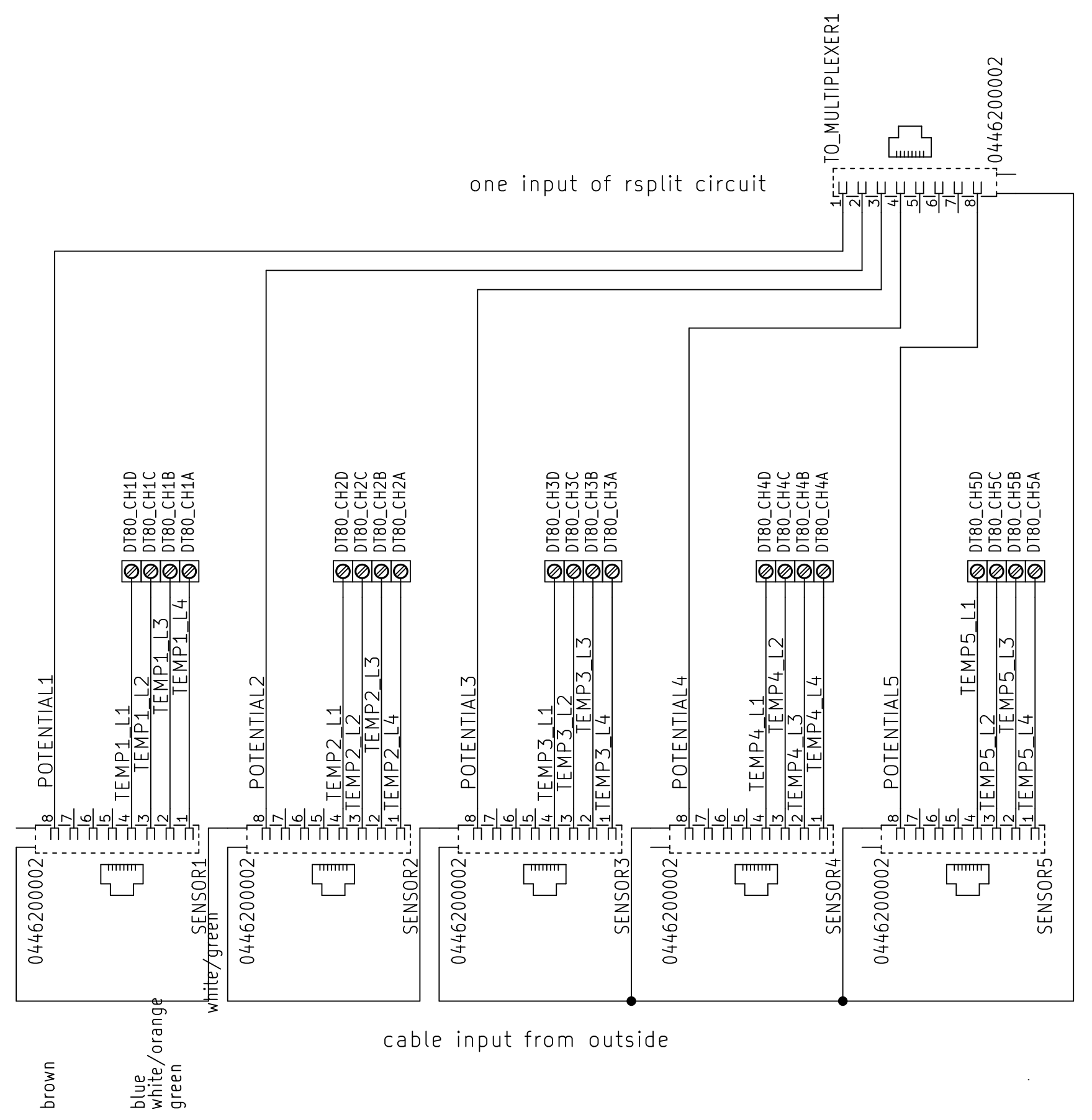

Figure 3: Input splitter diagram, splitting temperature and SP signal lines. Electrode group signals arrive from the lightning protection circuit at the bottom and are then routed either directly to the corresponding logger inputs for temperature measurements (groups of four output in the center), or, in groups of 5 SP signals, are routed to the resistance split circuit (ethernet output at the top). This circuit was duplicated four time to accommodate the 20 electrode groups. 


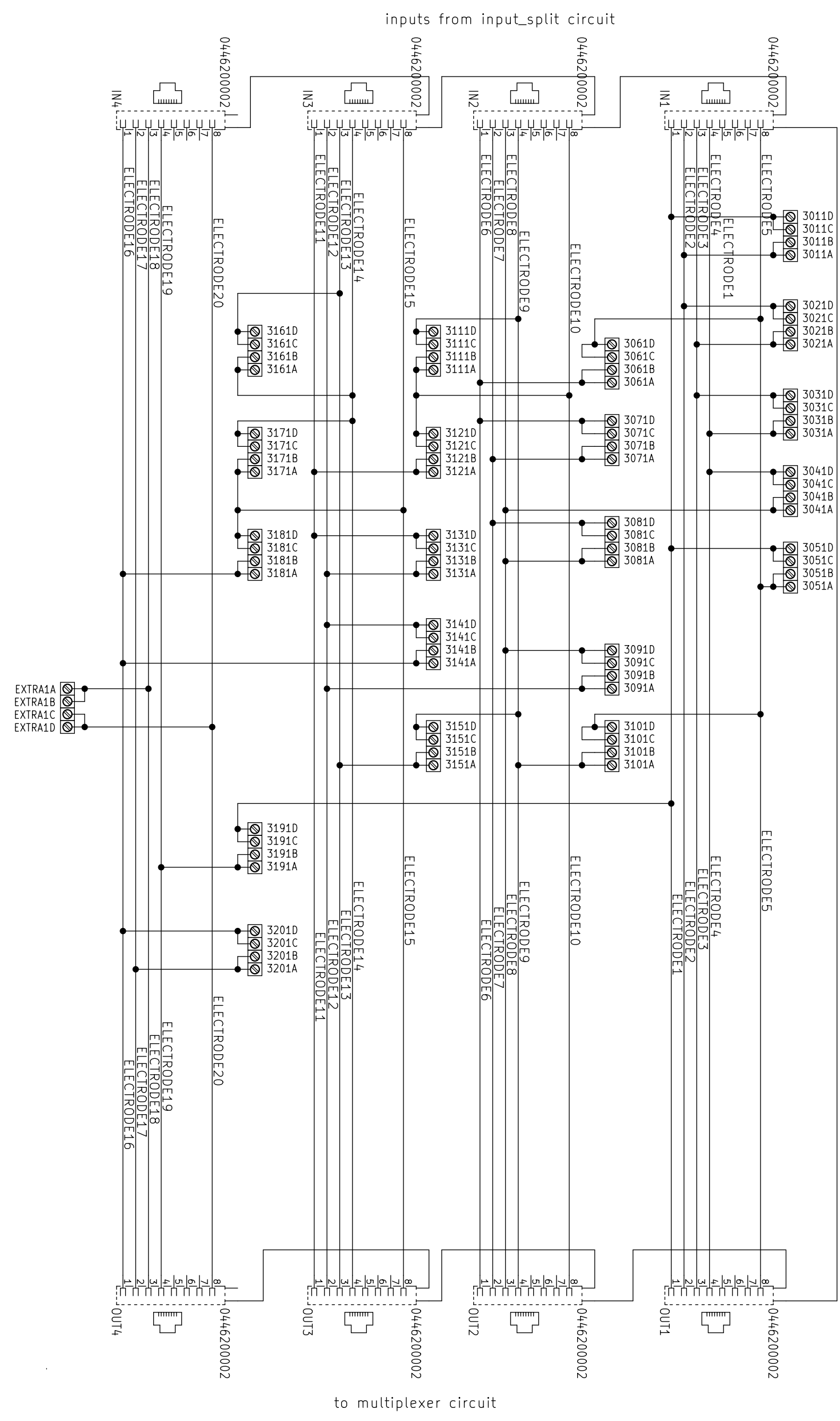

Figure 4: Resistance multiplexer diagram, multiplexing electrode lines to separate inputs for resistance measurements. SP signals arrive at the top from the four resistance splitting circuits. Signals are then multiplexed to certain logger inputs for resistance measurements (outputs in the center of the circuit, note that two outputs are always short circuited). The SP signals are directly routed to the outputs at the bottom for further multiplexing in the multiplexing circuit. 


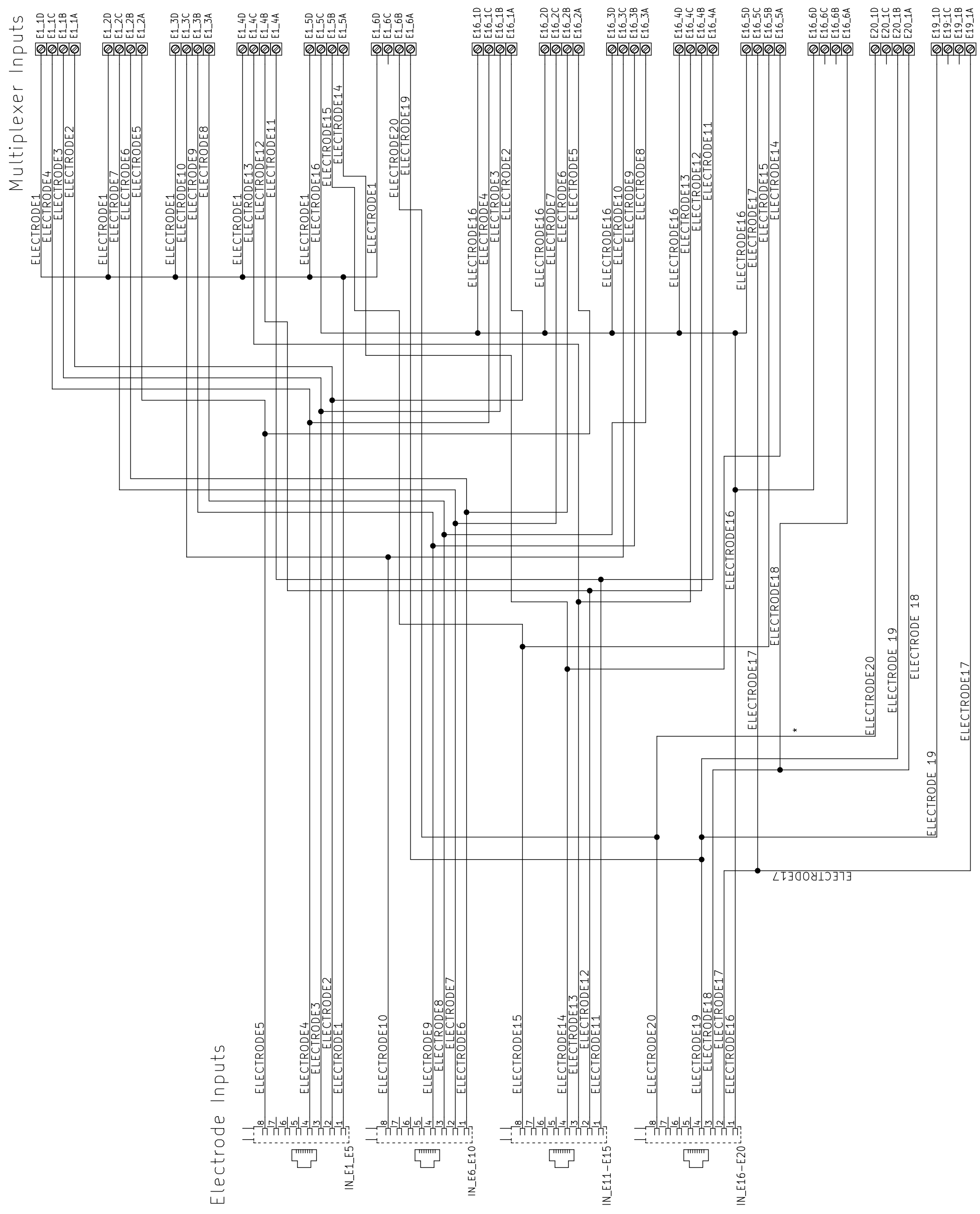

Figure 5: Multiplexer circuit diagram multiplexing SP input lines to specific inputs of the data logger. Each electrode is connected to one port on the bottom ("Electrode inputs") and then routed to one or more of the data logger multiplexer channels on the top ("Multiplexer Inputs"). Note that each logger input channel consists of four physical inputs, allowing for a variety of measurement combinations. 


\section{S3: Electrode matching}

before selection

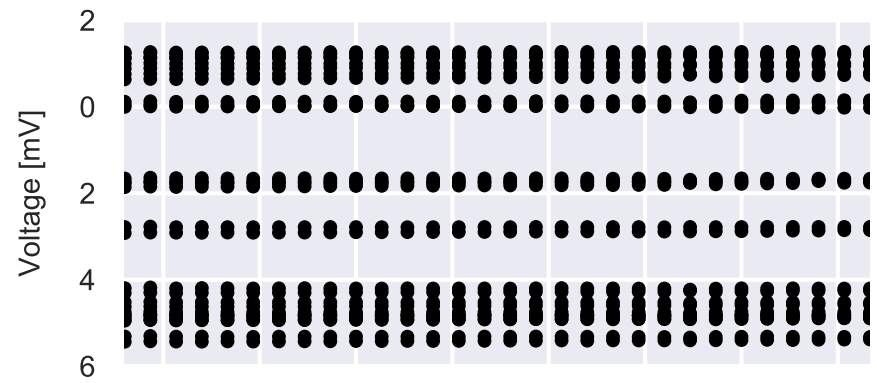

after selection

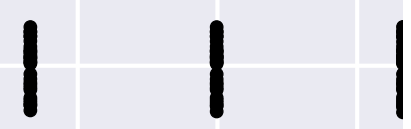
15.50
15.15
$16.00 \quad, 6.25$
$165^{50}$
$16 .^{15} \quad 17^{.00} \quad 1^{1.25}$
time [hours]
21.20
21.25
$2^{1.30}$
$2^{1.35}$
$2^{1.40}$
$21^{1.5}$
time [hours]

Figure 6: Test measurements of base-line electrical offsets in saline solution. Left: Voltage measurements for 20 randomly selected SP electrodes, right: Voltage measurements for 20 matched SP electrodes

The absolute potentials of SP electrodes changes with age, chemical environment, and usage history. As such, it is commonly advised to check the baseline potential difference between electrodes before measurements commence (e.g., Corwin, 1989), in order to not confuse the offsets with process-based signals. Out of the pool of available SP electrodes, we initially selected 20 random electrodes. This yielded voltages with respect to electrodes 1 and 16 of down to $-5.4 \mathrm{mV}$ (Fig. 6, left). Following this, electrodes were selected out of the available pool to minimize this offset voltage, resulting in a maximum voltage difference of $\pm 1 \mathrm{mV}$ (Fig. 6 , right). 


\section{S4: SP data filtering}
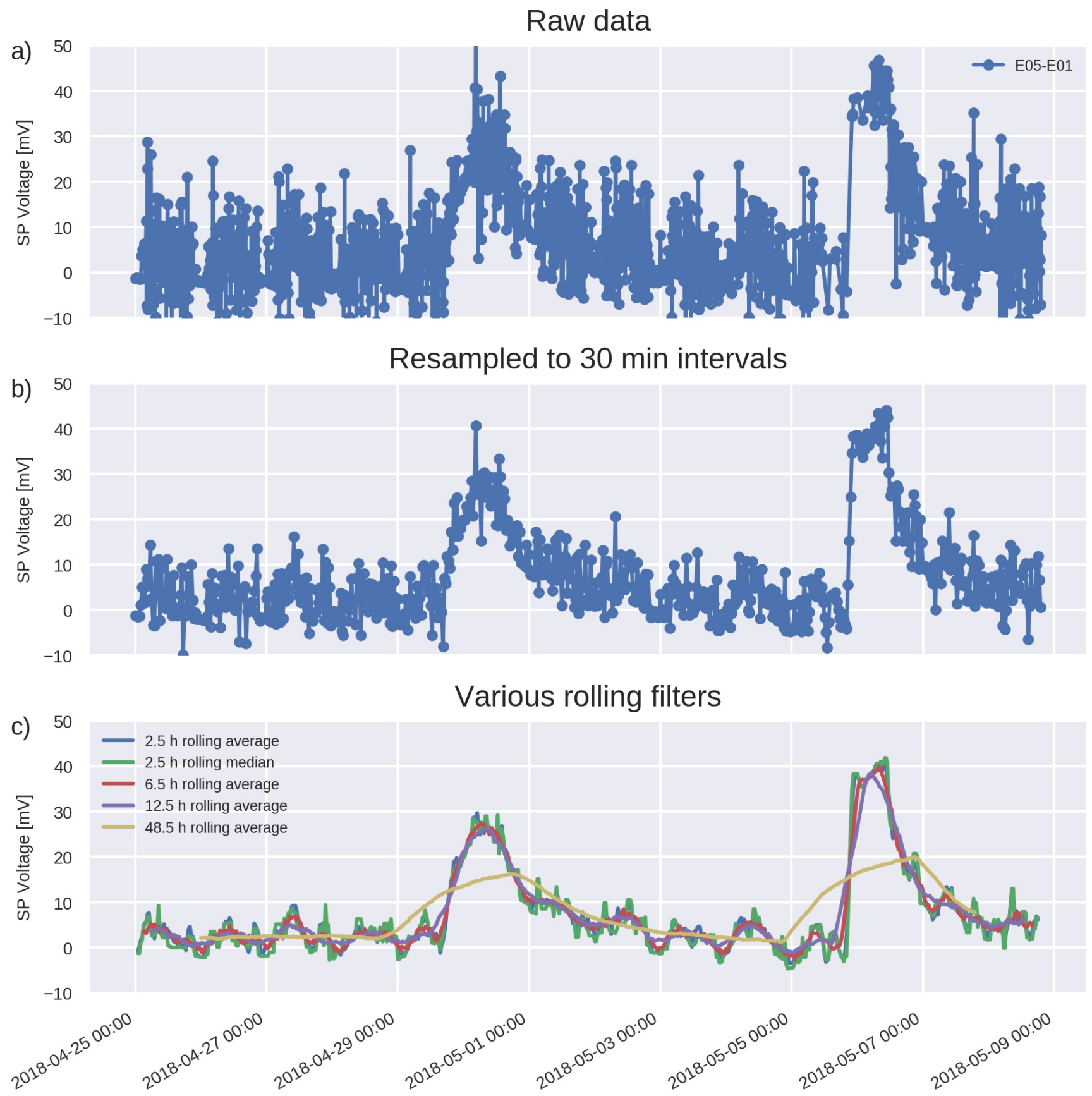

Figure 7: Data processing steps applied to SP data presented in this study, shown for the dipole E5-E1.

Captured SP data presented in this study exhibits quite large noise levels (e.g., Fig. 7a). This is caused by aliasing artifacts, as well as anthropogenic noise due to the operation of the cable-car to the summit and corresponding touristic operations. As such data was processed as follows: First, data was resampled to 30 minute intervals, using linear interpolation and averaging. The effect of this operation is exemplarily shown in Fig. 7b. Depending on the specific application, in the following various rolling mean filters are 
applied to the data, serving as simple, yet crude, low-pass filters. The effects of various window sizes is presented in Fig. $7 c$.

We fully acknowledge that the use of rolling average filters is not necessarily optimal for frequencydomain filtering. In addition, other filters, such as the rolling median or the alpha-trimmed-mean filter (Gersztenkorn and Scales, 1988), are known to better preserve transient spikes. Yet, in the present study we only aimed at presenting certain characteristics or concepts, which allowed us to use the robust and simple rolling average filter. 


\section{S5: Wavelet analysis of noise data}

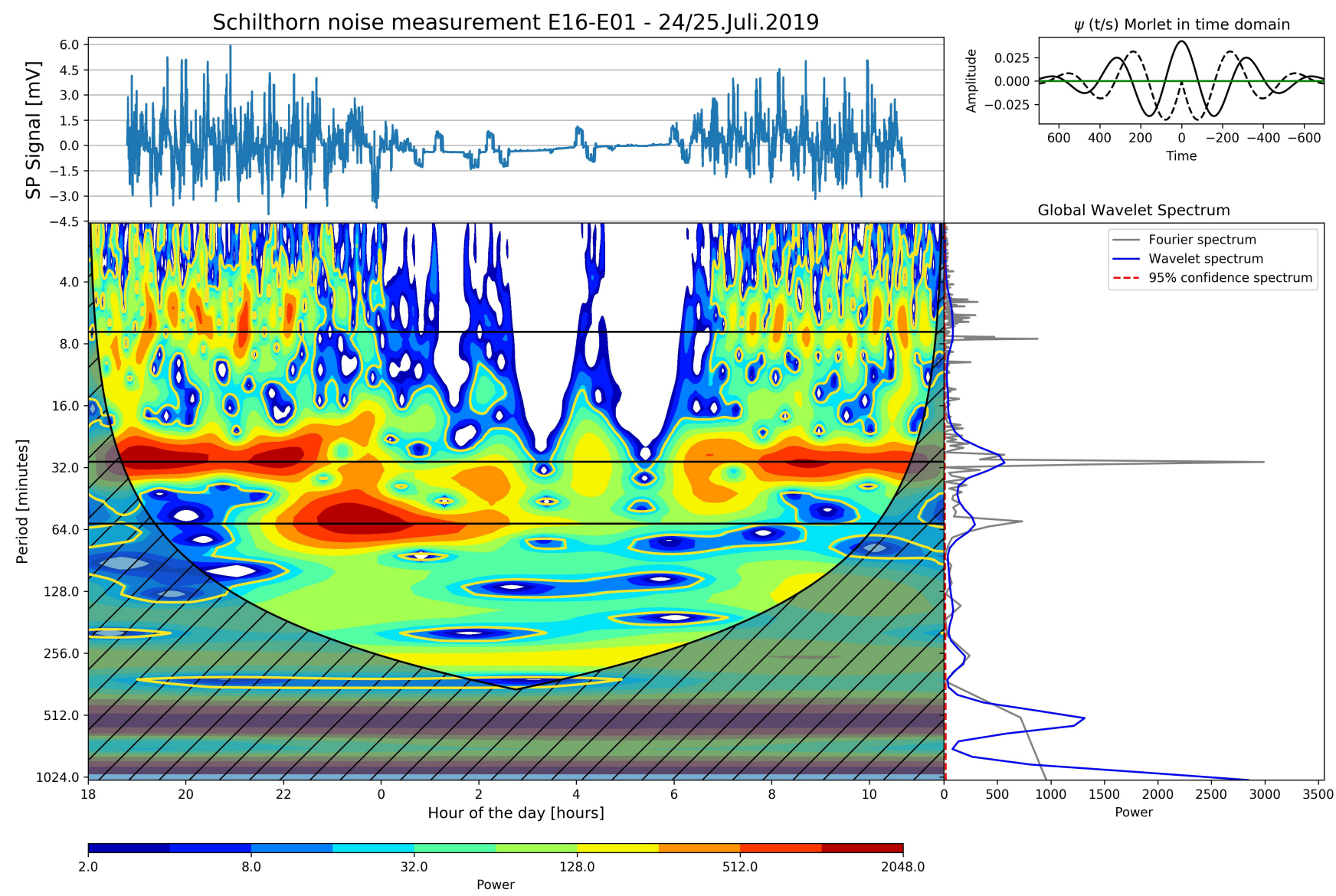

Figure 8: Wavelet analysis of the noise measurements 2019

In order to gain more information on spatio-temporal spectral content, a wavelet-based analysis (e.g., Grinsted et al., 2004) was conducted on the noise measurements conducted in 2019. The analysis shows strong 30 minute components during the day, a section of a few hours with 60 minute components just before night, and no significant spectral components during night-time hours (Fig. 8).

The analysis was conducted using the waipy software package (originally from https://github.com/ mabelcalim/waipy, fork used here: https://github.com/m-weigand/waipy). 


\section{Data Logger programming}

' DT80M program Schilthorn SP system 2017

' reset the logger

session stop

session clear

cevtlog

cerrlog

deld job $=*$ archive $=y$

deljob *

restore settings to default

factorydefaults

some debug infos

profile parameters $P 56=1$

we operate on UTC

profile locale time_zone $=+\mathrm{OH}$

sim:

profile modem service=gsm_preferred

profile modem MIN_SIGNAL_FOR_DATA_DBM=-110

' coop mobile $\mathrm{CH}$ internet settings

profile modem pin=XXXX

profile modem apn=click

profile modem apn_account=" "

profile modem apn_password=""

profile modem_session smtp_server $=\mathrm{XXXX}$

profile modem_session smtp_account $=\mathrm{XXXX}$

profile modem_session smtp_password=XXXX

PROFILE UNLOAD FTP_RETRIES $=10$

mains frequency $[\mathrm{Hz}]$

profile parameters $\mathrm{p} 11=20$

' sleep while on power, but not if USB is connected profile parameters $\mathrm{p} 15=1$

$5 \mathrm{~V}$ power output while the measurement system is on PWR5V $=1$

' should prevent some debug messages

profile http_server enable_wdg=no

reduce power by disabling ethernet

profile ethernet enable=no

profile ethernet ip_address $=192.168 .21 .10$

profile ethernet subnet_mask $=255.255 .255 .0$

' define functions for the yellow button

profile function f1_label = "mail data"

profile function $f 1$ _command = "copyd start=new $\backslash$

dest=mailto : XX@XX.XX?\&subject=sch17_01\&priority=high\&interface=modem \}

format $=\mathrm{dbd}$ merge $=\mathrm{N}^{\prime \prime}$

profile function f2_label = "PWR5V"

profile function $f 2$ _command $=$ "PWR5V=1" 
profile function f3_label $=$ "session signal"

profile function $f 3$ _command = "session signal"

profile function f4_label $=$ "download all"

profile function f4_command = "COPYD job=* dest=a: merge=N format=dbd;

servicedata a: $\backslash \backslash \backslash$ servicedata.txt; removemedia"

profile function f5_label $=$ "servicedata to usb"

profile function f5_command = "SERVICEDATA a:\\\servicedata.txt"

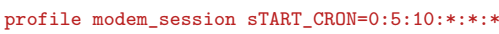

profile modem_session stop_CRON $=0: 20: 10: *: *: *$

profile modem_session timing_control=cron

' [ seconds : minutes : hours : day : month : weekday ]

BEGIN "sch17_01"

' measure voltages

RA"SPV" ("B: ", data : ov : 370D) 10M

$101 * \mathrm{~V}$ ("E02-E01", ES10)

$101+\mathrm{V}($ "E03-E01", ES10)

101-V ("E04-E01", ES10)

$102 * \mathrm{~V}(" \mathrm{E} 05-\mathrm{E} 01 ", \mathrm{ES} 10)$

102+V ("E06-E01", ES10)

102-V("E07-E01" , ES10)

103*V("E08-E01", ES10)

103+V ("E09-E01", ES10)

103-V("E10-E01", ES10)

$104 * \mathrm{~V}$ ("E11-E01" ,ES10)

104+V("E12-E01", ES10)

104-V ("E13-E01", ES10)

105*V ("E14-E01", ES10)

105+V ("E15-E01",ES10)

105-V("E16-E01" , ES10)

106*V ("E19-E01" , ES10)

106+V ("E20-E01" , ES10)

107*V ("E02-E16" , ES10)

107+V ("E03-E16" , ES10)

107-V ("E04-E16" ,ES10)

108*V ("E05-E16" ,ES10)

108+V ("E06-E16" , ES10)

108-V ("E07-E16" , ES10)

109*V ("E08-E16" , ES10)

109+V ("E09-E16" , ES10)

109-V ("E10-E16" , ES10)

110*V ("E11-E16" , ES10)

110+V("E12-E16" , ES10)

110-V ("E13-E16", ES10)

$111 * \mathrm{~V}$ ("E14-E16", ES10)

111+V ("E15-E16" , ES10)

111-V("E17-E16", ES10)

112*V ("E18-E16" , ES10)

113*V("E18-E20", ES10)

113+V ("E19-E20" ,ES10)

114*V("E17-E19",ES10)

$115 *$ HV ("Battery" )

116PT385 ("TempOutside" ,4W)

$117 * H V$ ("RH_INSIDE")

118*HV ("RH_OUTSIDE")

$119 *$ HV ("RAIN")

$120 * H V("$ SOLAR") 
' measure temperatures

RB"TEMPS" ("B: " , data : ov : 370D) $1 \mathrm{H}$

201*V("T1Voltage") 201PT385("Temp01", 4W)

202*V("T2Voltage") 202PT385("Temp02", 4W)

203*V("T3Voltage") 203PT385 ("Temp03", 4W)

204*V("T4Voltage") 204PT385 ("Temp04", 4W)

205*V("T5Voltage") 205PT385("Temp05" , 4W)

206*V("T6Voltage") 206PT385 ("Temp06", 4W)

207*V("T7Voltage") 207PT385("Temp07", 4W)

208*V ("T8Voltage") 208PT385 ("Temp08", 4W)

209*V ("T9Voltage") 209PT385 ("Temp09", 4W)

210*V("T10Voltage") 210PT385("Temp10",4W)

211*V("T11Voltage") 211PT385("Temp11", 4W)

212*V("T12Voltage") 212PT385("Temp12", 4W)

213*V("T13Voltage") 213PT385 ("Temp13", 4W

214*V("T14Voltage") 214PT385("Temp14", 4W)

215*V("T15Voltage") 215PT385("Temp15", 4W)

216*V("T16Voltage") 216PT385 ("Temp16", 4W)

217*V("T17Voltage") 217PT385("Temp17",4W)

218*V("T18Voltage") 218PT385("Temp18",4W)

219*V("T19Voltage") 219PT385 ("Temp19",4W)

220*V("T20Voltage") 220PT385("Temp20",4W)

REFT 1REFT 2REFT 3REFT

' Resistances

RC"RES" ("B: ", data: ov: 1MB) [0:5:6]

301R("R01-02" , III) 301*V("V01-02")

302R ("R02-03", III) 302*V("V02-03")

303R ("R03-04", III) 303*V("V03-04")

304R("R04-08", III) 304*V("V04-08")

305R ("R01-05", III) 305*V("V01-05")

306R("R05-06", III) 306*V ("V05-06")

307R("R06-07", III) 307*V("V06-07")

308R("R07-08", III) 308*V("V07-08")

309R ("R08-12", III) 309*V("V08-12")

310R ("R05-09", III) 310*V("V05-09")

311R ("R09-10", III) 311*V("V09-10")

312R("R10-11", III) 312*V("V10-11")

313R("R11-12", III) 313*V("V11-12")

$314 \mathrm{R}$ ("R12-16", III) $314 * \mathrm{~V}$ ("V12-16")

$315 R(" R 09-13 "$, III) $315 * V$ ("V09-13")

316R("R13-14", III) 316*V("V13-14")

$317 R$ ("R14-15", III) $317 * V$ ("V14-15")

318R("R15-16", III) 318*V("V15-16")

319R("R01-19", III) 319*V("V01-19")

320R("R16-17", III) 320*V("V16-17")

VLITH

R100

VREF

VZERO

at night we increase measurement intervals

$\operatorname{RD}[0: 5: 20] \quad \mathrm{IF}(115 * \mathrm{HV}>12.6)\{\mathrm{RA} 30 \mathrm{M}\}$

$\operatorname{RE}[0: 5: 4] \operatorname{IF}(115 * \mathrm{HV}>12.6)\{\mathrm{RA} 10 \mathrm{M}\}$

RF6H

DO $\{$ copyd start=new2 dest=mailto:XXX@XXX.XX?\&

subject $=$ sch17_01\&priority=high\&interface $=$ modem format $=$ dbd merge $=\mathrm{N}\}$ 


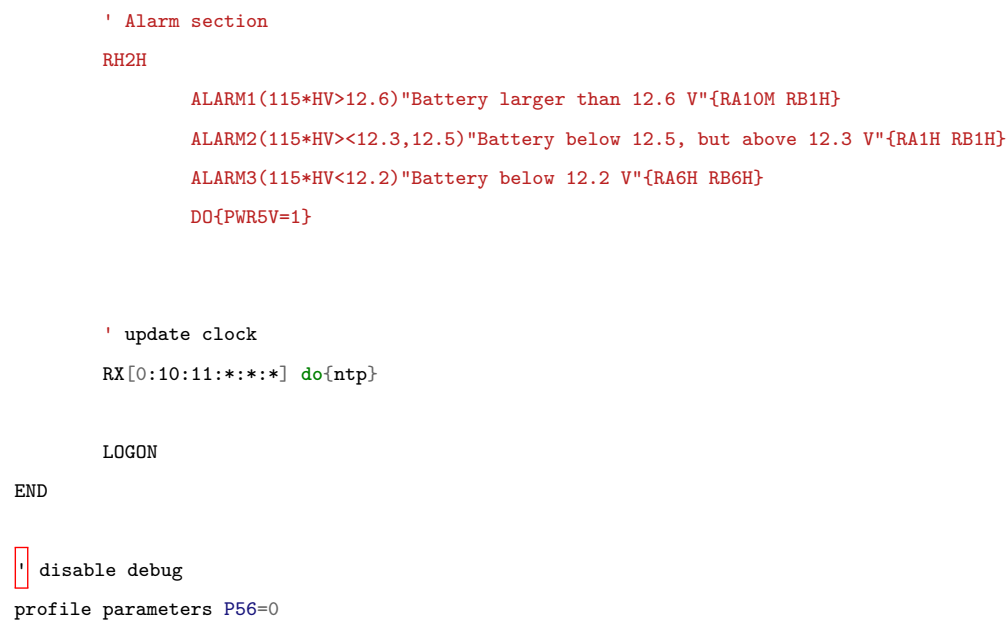

Listing 1: Programming of the DataTaker DT8OM - Series 4 logger 


\section{S6: Laboratory Experiment}

(a)

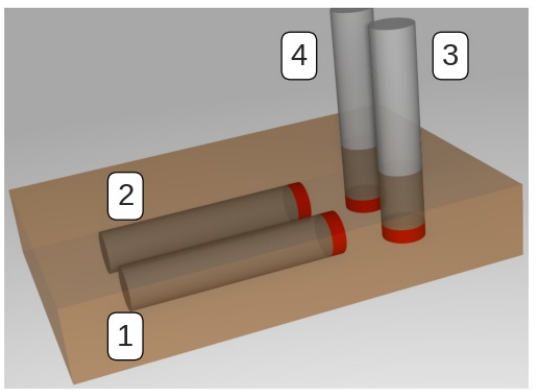

(b)

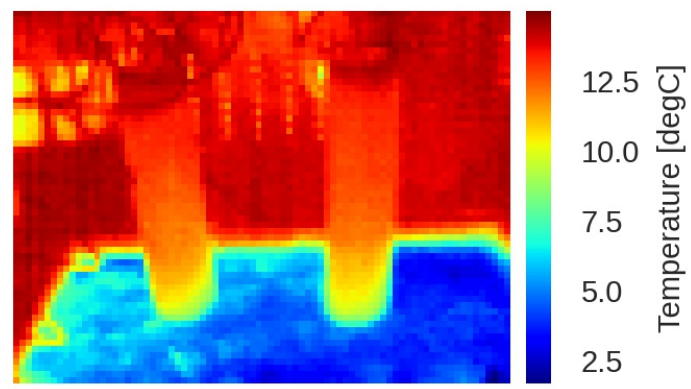

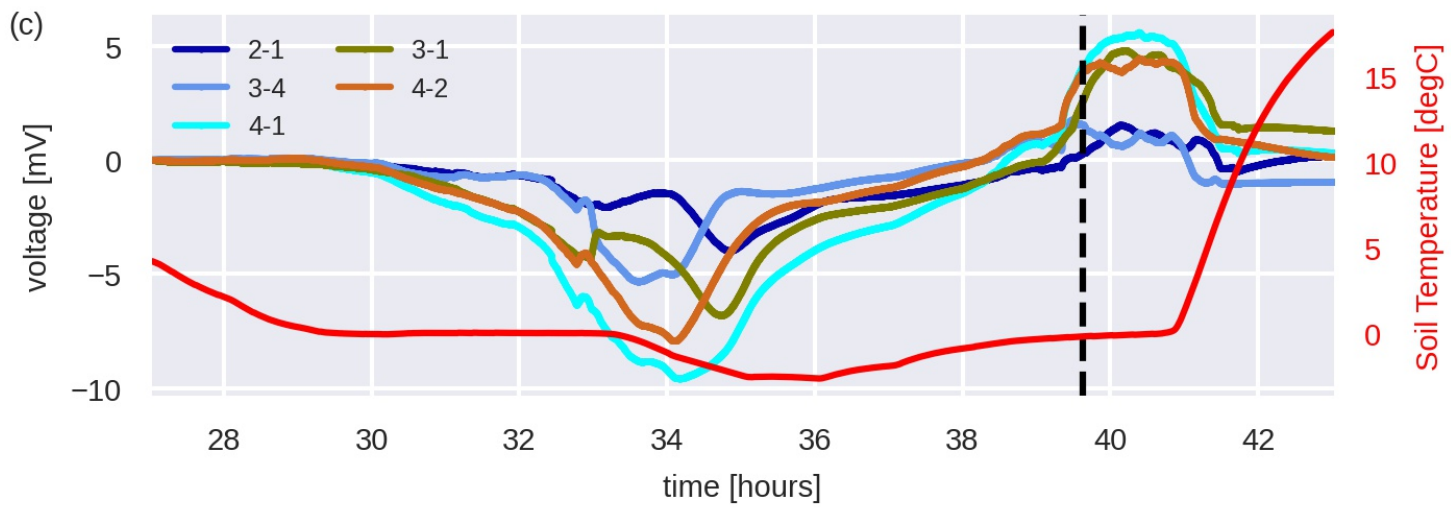

Figure 9: Laboratory experiment investigating electrode effects during freezing and thawing. a) Sketch of experimental setup. The container is $30 \mathrm{~cm}$ in length, $20 \mathrm{~cm}$ in depth, and $5 \mathrm{~cm}$ high. Electrodes are shown in gray, with the wood membranes in contact with the surrounding highlighted in red and assigned electrode numbers in white boxes. b) Thermal image (surface temperatures) during thawing c) Electrical voltages and soil temperatures; vertical black line indicates time of thermal image in b). 


\section{References}

Corwin, R.: Data quality for engineering self-potential surveys, in: Detection of Subsurface Flow Phenomena, edited by Merkler, G.-P., Militzer, H., Hötzl, H., Armbruster, H., and Brauns, J., pp. 49-72, Springer Berlin Heidelberg, Berlin, Heidelberg, https://doi.org/10.1007/BFb0011630, 1989.

Gersztenkorn, A. and Scales, J. A.: Smoothing seismic tomograms with alpha-trimmed means, Geophysical Journal International, 92, 67-72, https://doi.org/10.1111/j.1365-246X.1988.tb01121.x, 1988.

Grinsted, A., Moore, J., and Jevrejeva, S.: Application of the cross wavelet transform and wavelet coherence to geophysical time series, Nonlinear processes in geophysics, 11, 561-566, https://doi.org/10.5194/npg-11-561-2004, 2004. 